\title{
EXTENDING THE $t$-DESIGN CONCEPT
}

\author{
A. R. CALDERBANK AND P. DELSARTE
}

\begin{abstract}
Let $\mathfrak{B}$ be a family of $k$-subsets of a $v$-set $V$, with $1 \leq k \leq v / 2$. Given only the inner distribution of $\mathfrak{B}$, i.e., the number of pairs of blocks that meet in $j$ points (with $j=0,1, \ldots, k$ ), we are able to completely describe the regularity with which $\mathfrak{B}$ meets an arbitrary $t$-subset of $V$, for each order $t$ (with $1 \leq t \leq v / 2$ ). This description makes use of a linear transform based on a system of dual Hahn polynomials with parameters $v, k, t$. The main regularity parameter is the dimension of a well-defined subspace of $\mathbb{R}^{t+1}$, called the $t$-form space of $\mathfrak{B}$. (This subspace coincides with $\mathbb{R}^{t+1}$ if and only if $\mathfrak{B}$ is a $t$-design.) We show that the $t$-form space has the structure of an ideal, and we explain how to compute its canonical generator.
\end{abstract}

\section{INTRODUCTION}

Consider a set (a design) $\mathfrak{B}$ whose elements, called blocks, are $k$-subsets of a given $v$-set $V$ of points, with $1 \leq k \leq v / 2$. For a given integer $t$, in the range $0 \leq t \leq k$, we are interested in some regularity properties of $\mathfrak{B}$, of order $t$, which can be defined as follows. With any $t$-subset $x^{t}$ of $V$ and any integer $i \in[0, t]$ we associate the number $D_{i}\left(x^{t}\right)$ that counts the blocks in $\mathfrak{B}$ meeting $x^{t}$ in $t-i$ points. Suppose that we have a linear relation

$$
f_{0} D_{0}\left(x^{t}\right)+f_{1} D_{1}\left(x^{t}\right)+\cdots+f_{t} D_{t}\left(x^{t}\right)=c,
$$

where $f_{0}, f_{1}, \ldots, f_{t}$ and $c$ are fixed real numbers. Then we say that the $(t+1)$-tuple $\left(f_{i}\right)_{i=0}^{t}$ is a $t$-form for $\mathfrak{B}$. The set of $t$-forms clearly is a vector space, which will be called the $t$-form space of $\mathfrak{B}$. We propose to take the dimension of that space as a measure of the regularity of $\mathfrak{B}$ with respect to the $t$-subsets of $V$.

The idea originates mainly from a recent paper [2] in which linear relations as above, with the restrictive property $f_{1} \neq 0, f_{2}=\cdots=f_{t}=0$, are deduced from a strengthening of the Assmus-Mattson theorem in coding theory. This investigation has been continued in a companion paper [1]. Recall that $\mathfrak{B}$ is said to be a $t$-design, of index $c$, when $D_{0}\left(x^{t}\right)=c$ for all $t$-subsets $x^{t}$ of $V$. (For a thorough algebraic study, see especially [13].) This implies that every $(t+1)$-tuple $\left(f_{i}\right)_{i=0}^{t}$ is a $t$-form for $\mathfrak{B}$. So, our definition of regularity can be viewed as an extension of the classical $t$-design concept.

Received by the editors November 3, 1989 and, in revised form, May 20, 1991.

1991 Mathematics Subject Classification. Primary 05B20, 05B30; Secondary 33A63, 20 C30.

$K e y$ words and phrases. $t$-design, $t$-form space, $t$-distribution matrix, dual Hahn polynomials. 
We shall see how the theory of $t$-form spaces provides us with a satisfactory "combinatorial interpretation" of the algebraic notion of a $T$-design [3, 4], with $T$ a subset of $[0, t]$. One of the main results of this paper is an explicit description of the $t$-form space in terms of the inner distribution of $\mathfrak{B}$. In fact, this space is proved to be an ideal of a well-defined $(t+1)$-dimensional commutative algebra, and to admit a canonical generator which can be computed from the inner distribution. Furthermore, the theory gives us an efficient method to determine the $t$-distribution matrix of $\mathfrak{B}$, having the numbers $D_{i}\left(x^{t}\right)$ as its entries, from a minimum set of data. The methods of proof are based on a suitable matrix transform, involving a system of dual Hahn polynomials (with parameters $v, k, t)[6,7,10]$. Some combinatorial applications of the Hahn polynomials (quite different from those considered in what follows) can be found in [4, 12, and 13].

For several reasons, it is useful to replace the "combinatorial" notion of a design $\mathfrak{B}$ by the more general "algebraic" notion of a design vector $\boldsymbol{\beta}$, which is defined simply as a real row vector, of size $\left(\begin{array}{l}v \\ k\end{array}\right)$, with components labelled by the $k$-subsets of $V$ (see [4], for example). The concept of the $t$-form space can be extended naturally to this general definition of a design vector. (The situation described above corresponds to the special case where $\boldsymbol{\beta}$ is the characteristic vector of a combinatorial design $\mathfrak{B}$.) All our theorems are valid for general design vectors. Note that the theory would be essentially the same if we would consider only rational vectors $\boldsymbol{\beta}$ or, equivalently, integer vectors $\boldsymbol{\beta}$, i.e., signed designs $[8,9,11]$.

\section{2. $t$-FORMS AND THE $t$-DISTRIBUTION MATRIX}

Let $V$ be a $v$-set (i.e., a set of finite cardinality $v$ ), and let $\mathscr{P}^{i}$ be the set of $i$ subsets of $V$, for $i=0,1, \ldots, v$. Given an integer $k$, with $0 \leq k \leq\lfloor v / 2\rfloor$, we shall be interested in the real $\left(\begin{array}{l}v \\ k\end{array}\right)$-dimensional vector space $\mathbb{R} \mathscr{P}^{k}$, with entries indexed by the elements $x^{k}$ of $\mathscr{P}^{k}$. (In this paper, we always use row vectors.) Consider a vector $\boldsymbol{\beta}$ in $\mathbb{R}^{k}$. If $\beta\left(x^{k}\right)=0$ or 1 for every $x^{k}$, then we can identify $\boldsymbol{\beta}$ with the set $\mathfrak{B}=\left\{x^{k} \in \mathscr{P}^{k}: \beta\left(x^{k}\right)=1\right\}$ that has $\boldsymbol{\beta}$ as its characteristic vector. Such a subset $\mathfrak{B}$ of $\mathscr{P}^{k}$ is a "design" for the Johnson scheme $J(v, k)$; the elements of $\mathfrak{B}$ are often called blocks. By extension, we shall say that any vector $\boldsymbol{\beta}$ in $\mathbb{R} \mathscr{P}^{k}$ is a design vector for $J(v, k)$.

Let $t$ be a fixed integer, with $t \in[0, k]$. We shall express the "regularity properties of order $t$ " of a given design vector $\boldsymbol{\beta}$ through the notions of the $t$-distribution matrix and the $t$-form space.

Definition 2.1. For $i \in[0, t]$, the $i$ th adjacency matrix of $\mathscr{P}^{k}$ with respect to $\mathscr{P}^{t}$ is the $\{0,1\}$ matrix $C_{i}^{k, t}$, of size $\left(\begin{array}{l}v \\ k\end{array}\right) \times\left(\begin{array}{l}v \\ t\end{array}\right)$, with rows indexed by $x^{k} \in \mathscr{P}^{k}$ and columns indexed by $x^{t} \in \mathscr{P}^{t}$, defined by

$$
C_{i}^{k, t}\left(x^{k}, x^{t}\right)=1 \text { if and only if }\left|x^{k} \cap x^{t}\right|=t-i .
$$

The $t$-distribution matrix of the design vector $\boldsymbol{\beta} \in \mathbb{R} \mathscr{P}^{k}$ is the real matrix $D^{t}$, of size $(t+1) \times\left(\begin{array}{l}v \\ t\end{array}\right)$, with $i$ th row

$$
D_{i}^{t}=\boldsymbol{\beta} C_{i}^{k, t}, \quad \text { for } i \in[0, t] .
$$


The combinatorial meaning of this definition is the following. If $\boldsymbol{\beta}$ is the characteristic vector of a subset $\mathfrak{B}$ of $\mathscr{P}^{k}$, then the $\left(i, x^{t}\right)$ entry $D_{i}^{t}\left(x^{t}\right)$ of the $t$-distribution matrix of $\boldsymbol{\beta}$ counts the blocks in $\mathfrak{B}$ that have $t-i$ points in common with $x^{t}$. (Note that the $k$-distribution matrix $D^{k}$ coincides with the "outer distribution matrix" in [3].)

Definition 2.2. A vector $\mathbf{f}^{t}=\left(f_{0}^{t}, f_{1}^{t}, \ldots, f_{t}^{t}\right)$ in $\mathbb{R}^{t+1}$ is a $t$-form for the design vector $\boldsymbol{\beta} \in \mathbb{R} \mathscr{P} k$ if and only if it satisfies

$$
\mathbf{f}^{t} D^{t}=c \mathbf{1}, \text { with } c \in \mathbb{R},
$$

where 1 denotes the all-one vector. The subspace of $\mathbb{R}^{t+1}$ formed by all the $t$-forms for $\boldsymbol{\beta}$ will be denoted by $\mathscr{F} t$ and will be referred to as the $t$-form space of $\boldsymbol{\beta}$. Finally, the $t$-degree of $\boldsymbol{\beta}$, denoted by $r^{t}$ (or simply by $r$ ), is defined as the codimension of the $t$-form space, that is,

$$
r^{t}=r=t+1-\operatorname{dim}\left(\mathscr{F}^{t}\right) .
$$

By definition, $\mathbf{f}^{t}$ is a $t$-form iff the linear combination $\sum_{i=0}^{t} f_{i}^{t} D_{i}^{t}\left(x^{t}\right)$ is constant over the set $\mathscr{P} t$. It seems therefore natural to consider that a design vector $\boldsymbol{\beta}$ is highly regular with respect to $\mathscr{P} t$ when it admits a large number of linearly independent $t$-forms, i.e., when its $t$-degree $r^{t}$ is small. One may say that $r^{t}$ is a measure of the " $t$-irregularity" of $\boldsymbol{\beta}$. The most regular designs $\mathfrak{B}$ are the $t$-designs (in the usual sense), defined by the fact that $D_{0}^{t}\left(x^{t}\right)$ is a constant. This implies that $D_{i}^{t}\left(x^{t}\right)$ is a constant, for each $i \in[0, t]$. Therefore, a $t$-design $\mathfrak{B}$ is characterized by the fact that its $t$-form space coincides with $\mathbb{R}^{t+1}$, i.e., by the property $r^{t}=0$.

Let us now emphasize the simple relationship between the $t$-degree $r^{t}$ and the rank of $D^{t}$. (In what follows, we denote by $\langle\boldsymbol{\xi}, \boldsymbol{\eta}\rangle$ the inner product $\boldsymbol{\xi}^{T}$ of two vectors $\boldsymbol{\xi}$ and $\boldsymbol{\eta}$ in $\mathbb{R} \mathscr{P}^{k}$.) We need a preliminary result.

Lemma 2.1. The constant $c$ in the equation $\mathbf{f}^{t} D^{t}=c \mathbf{1}$ is determined from $\mathbf{f}^{t}$ and $\langle\boldsymbol{\beta}, \mathbf{1}\rangle$ by

$$
c\left(\begin{array}{l}
v \\
t
\end{array}\right)=\langle\boldsymbol{\beta}, \mathbf{1}\rangle \sum_{i=0}^{t} f_{i}^{t}\left(\begin{array}{c}
k \\
t-i
\end{array}\right)\left(\begin{array}{c}
v-k \\
i
\end{array}\right) .
$$

Proof. We have $D_{i}^{t} \mathbf{1}^{T}=\langle\boldsymbol{\beta}, \mathbf{1}\rangle\left(\begin{array}{c}k \\ t-i\end{array}\right)\left(\begin{array}{c}v-k \\ i\end{array}\right)$, by simple counting of $t$-sets relative to a fixed $k$-set. Using this identity, we prove the lemma by computing the inner product of both sides of $\mathbf{f}^{t} D^{t}=c \mathbf{1}$ with 1 .

Proposition 2.1. The t-form space $\mathscr{F}$ t of any design vector $\boldsymbol{\beta}$ contains the allone vector $\mathbf{1}$, and the t-distribution matrix $D^{t}$ of $\boldsymbol{\beta}$ has rank $r^{t}$ or $r^{t}+1$ according as $\langle\boldsymbol{\beta}, \mathbf{1}\rangle=0$ or $\langle\boldsymbol{\beta}, \mathbf{1}\rangle \neq 0$.

Proof. The first part is obvious, for $\mathbf{1} D^{t}=\langle\boldsymbol{\beta}, \mathbf{1}\rangle \mathbf{1}$. The second part is proved as follows. If $\langle\boldsymbol{\beta}, \mathbf{1}\rangle=0$, then any $t$-form equation $\mathbf{f}^{t} D^{t}=c \mathbf{1}$ must satisfy $c=0$, by Lemma 2.1, which means that $\mathscr{F}^{t}$ equals the orthogonal complement of the column space of $D^{t}$. On the other hand, if $\langle\boldsymbol{\beta}, \mathbf{1}\rangle \neq 0$, then we see that the $t$-form 1 is not orthogonal to the column space of $D^{t}$. 
Note that the distinction between the cases $\langle\boldsymbol{\beta}, \mathbf{1}\rangle=0$ and $\langle\boldsymbol{\beta}, \mathbf{1}\rangle \neq 0$ is inconsequential, since replacing $\boldsymbol{\beta}$ by $\boldsymbol{\beta}+a \mathbf{1}$, with any $a \in \mathbb{R}$, does not affect the $t$-form space.

\section{HaRmonic analysis and dual Hahn polynomials}

To determine the $t$-form space of a design vector we shall use a transform method involving dual Hahn polynomials. We need some preliminaries about the harmonic analysis of the symmetric group $S_{v}$ (see $[5,6,7]$ ). Under the natural action of $S_{v}$, the space $\mathbb{R}^{k}$ decomposes as the orthogonal sum of $k+$ 1 irreducible $S_{v}$-invariant subspaces, called the harmonic subspaces of $\mathbb{R}^{k}$. A "constructive definition" is given below.

Definition 3.1. For $s \in[0, k]$, the homogeneous subspace of degree $s$ of $\mathbb{R}^{k}$, denoted by $\operatorname{Hom}_{s}^{k}$, is defined as the row space of $\left(C_{0}^{k, s}\right)^{T}$ (which is the characteristic matrix of the inclusion relation between $s$-subsets and $k$-subsets of $V)$. The harmonic subspace of degree $s$ of $\mathbb{R}^{k}$ is denoted by $\mathrm{Harm}_{s}^{k}$ and is defined as the orthogonal complement of $\operatorname{Hom}_{s-1}^{k}$ in $\operatorname{Hom}_{s}^{k}$.

The latter definition makes sense since $\operatorname{Hom}_{s-1}^{k}$ is a subspace of $\mathrm{Hom}_{s}^{k}$, as a consequence of $C_{0}^{k, s} C_{0}^{s, s-1}=(k-s+1) C_{0}^{k, s-1}$. Note the properties $\operatorname{Hom}_{k}^{k}=\mathbb{R}^{k}$ and $\operatorname{Hom}_{0}^{0}=\operatorname{Harm}_{0}^{0}=\mathbb{R} \mathbf{1}$. As the columns of $C_{0}^{k, s}$ are linearly independent, $\operatorname{Hom}_{s}^{k}$ has dimension $\left(\begin{array}{l}v \\ s\end{array}\right)$ and $\operatorname{Harm}_{s}^{k}$ has dimension $\left(\begin{array}{l}v \\ s\end{array}\right)-\left(\begin{array}{c}v \\ s-1\end{array}\right)$.

Definition 3.2. Let $\mathscr{A}^{k, t}$ be the real $(t+1)$-dimensional vector space generated by the adjacency matrices $C_{i}^{k, t}$ with $i \in[0, t]$. In particular, $\mathscr{A}^{k, k}$ is the Bose-Mesner algebra of the Johnson scheme $J(v, k)$. For $s \in[0, k]$, let $E_{s}^{k}$ be the orthogonal projection matrix from $\mathbb{R} \mathscr{P} k$ onto $\mathrm{Harm}_{s}^{k}$. The matrices $E_{s}^{k}$ are the irreducible idempotents of $\mathscr{A}^{k, k}$.

Let us now give two useful results concerning, first, the relation between the spaces $\mathscr{A}^{k, t}$ and the algebra $\mathscr{A}^{k, k}$ and, next, the relation between the eigenspaces of both algebras $\mathscr{A}^{k, k}$ and $\mathscr{A}^{t, t}$.

Lemma 3.1. The space $\mathscr{A}^{k, t}$ is generated by the $t+1$ matrices $E_{s}^{k} C_{0}^{k, t}$ with $s \in[0, t]$.

Proof. A straightforward argument shows that if $X$ belongs to $\mathscr{A}^{k, k}$, then $X C_{0}^{k, t}$ belongs to $\mathscr{A}^{k, t}$. It remains to show that the matrices $E_{s}^{k} C_{0}^{k, t}$ are linearly independent. This follows from the well-known fact (easily deduced from the information above) that $C_{0}^{k, t}\left(C_{0}^{k, t}\right)^{T}$ is a linear combination of $E_{0}^{k}$, $E_{1}^{k}, \ldots, E_{t}^{k}$ with positive coefficients. More precisely, as shown in [3], the coefficient of $E_{i}^{k}$ is $\left(\begin{array}{c}k-i \\ k-t\end{array}\right)\left(\begin{array}{c}v-t-i \\ k-t\end{array}\right)$ for $i=0,1, \ldots, t$.

Lemma 3.2. For $s \in[0, t]$, the harmonic subspaces of degree $s$ of $\mathbb{R} \mathscr{P} k$ and $\mathbb{R} \mathscr{P} t$ are related by

$$
\operatorname{Harm}_{s}^{k}=\operatorname{Harm}_{s}^{t}\left(C_{0}^{k, t}\right)^{T}, \quad \operatorname{Harm}_{s}^{t}=\operatorname{Harm}_{s}^{k} C_{0}^{k, t} .
$$


Hence, the irreducible idempotents of the Bose-Mesner algebras $\mathscr{A}^{k, k}$ and $\mathscr{A}^{t, t}$ are related by $C_{0}^{k, t} E_{s}^{t}=E_{s}^{k} C_{0}^{k, t}$.

Proof. We start from $C_{0}^{k, t} C_{0}^{t, s}=\left(\begin{array}{c}k-s \\ t-s\end{array}\right) C_{0}^{k, s}$. This shows that the first statement of the lemma is satisfied when Harm is replaced by Hom (in view of the fact that $C_{0}^{k, t}\left(C_{0}^{k, t}\right)^{T}$ preserves $\left.\mathrm{Hom}_{s}^{k}\right)$. The statement about harmonic spaces can then be deduced (by use of Definition 3.1) from the fact that $\mathrm{Harm}_{s}^{k}$ is an eigenspace of $C_{0}^{k, t}\left(C_{0}^{k, t}\right)^{T}$, with a positive eigenvalue. The second statement readily follows from the first one. Further details are omitted.

Next, we introduce a three-parameter family of dual Hahn polynomials [10] (with a normalization adapted to our subject), and recall some of their wellknown properties.

Definition 3.3. For $i \in[0, t]$, the dual Hahn polynomial $Q_{i}^{k, t}(z)$, with $0 \leq$ $t \leq k \leq\lfloor v / 2\rfloor$, is defined by

$$
\left(\begin{array}{c}
k \\
t
\end{array}\right) Q_{i}^{k, t}(z)=\left(\begin{array}{c}
k \\
t-i
\end{array}\right)\left(\begin{array}{c}
v-k \\
i
\end{array}\right) \sum_{l=0}^{i} \frac{(-i)_{l}(-z)_{l}(z-v-1)_{l}}{(1)_{l}(-t)_{l}(k-v)_{l}}
$$

where $(a)_{l}=a(a+1) \cdots(a+l-1)$. Thus, $Q_{i}^{k, t}(z)$ has degree $2 i$ in $z$ and degree $i$ in the variable $\lambda^{t}(z)=Q_{1}^{t, t}(z)=t(v-t)-z(v+1-z)$.

Theorem 3.1. The dual Hahn polynomials satisfy the following orthogonality relation and three-term recurrence relation:

$$
\begin{gathered}
\sum_{s=0}^{t} \frac{v-2 s+1}{v-s+1}\left(\begin{array}{c}
v \\
s
\end{array}\right)\left(\begin{array}{c}
k-s \\
k-t
\end{array}\right)\left(\begin{array}{c}
v-t-s \\
k-t
\end{array}\right) Q_{i}^{k, t}(s) Q_{j}^{k, t}(s) \\
=\left(\begin{array}{c}
v \\
k
\end{array}\right)\left(\begin{array}{c}
k \\
t-i
\end{array}\right)\left(\begin{array}{c}
v-k \\
i
\end{array}\right) \delta_{i, j}, \\
\lambda^{t}(z) Q_{i}^{k, t}(z)=\sum_{j=i-1}^{i+1} w_{i, j}^{k, t} Q_{j}^{k, t}(z),
\end{gathered}
$$

with $w_{i, i-1}^{k, t}=(t+1-i)(v+1-k-i), w_{i, i}^{k, t}=(t-i)(k-t+i)+i(v-k-i)$, $w_{i, i+1}^{k, t}=(i+1)(k+1-t+i)$, and $\lambda^{t}(z)=t(v-t)-z(v+1-z)$.

The fundamental reason why the dual Hahn polynomials occur in our investigation can be explained in a group theoretic setting by the fact that these polynomials give the spherical and intertwining functions involved in the harmonic analysis relative to the symmetric group [6,7]. Not surprisingly, the spherical case, $t=k$, plays a special role in our theory (see [5]). The following theorem shows, in explicit terms, how the dual Hahn polynomials $Q_{i}^{k, t}(z)$ appear on the scene.

Theorem 3.2. The adjacency matrices $C_{i}^{k, t}$ are related to the irreducible idempotents $E_{s}^{k}$ of the Bose-Mesner algebra of $J(v, k)$ by

$$
C_{i}^{k, t}=\left(\sum_{s=0}^{t} Q_{i}^{k, t}(s) E_{s}^{k}\right) C_{0}^{k, t}, \quad \text { for } i \in[0, t] .
$$


Proof. In view of Lemma 3.1, we may write the relation above if we replace $Q_{i}^{k, t}(s)$ by an unknown real number $X_{i}^{k, t}(s)$. To prove the desired coincidence, we start from the matrix identity

$$
C_{i}^{k, t} C_{1}^{t, t}=\sum_{j=0}^{t} w_{i, j}^{k, t} C_{j}^{k, t} .
$$

Here, $w_{i, j}^{k, t}$ counts the elements $y^{t}$ of $\mathscr{P}^{t}$ that satisfy $\left|x^{k} \cap y^{t}\right|=t-i$ and $\left|y^{t} \cap x^{t}\right|=t-1$, for a given pair $\left(x^{k}, x^{t}\right) \in \mathscr{P}^{k} \times \mathscr{P}^{t}$ with $\left|x^{k} \cap x^{t}\right|=t-j$. In particular, $w_{i, j}^{k, t}=0$ if $|i-j| \geq 2$. A counting argument shows that the intersection numbers $w_{i, j}^{k, t}$ thus defined (for $|i-j| \leq 1$ ) coincide with the recurrence parameters for the dual Hahn polynomials (see Theorem 3.1). Postmultiplying the identity above by $E_{s}^{t}$ (with $s \in[0, t]$ ) and using the last statement in Lemma 3.2, together with the well-known formula $C_{1}^{t, t} E_{s}^{t}=\lambda^{t}(s) E_{s}^{t}$, we readily obtain the three-term relation

$$
\lambda^{t}(s) X_{i}^{k, t}(s)=\sum_{j=i-1}^{i+1} w_{i, j}^{k, t} X_{j}^{k, t}(s) .
$$

This uniquely determines the unknowns $X_{i}^{k, t}(s)$, from the initial values $X_{0}^{k, t}(s)$ $=1$ and $X_{-1}^{k, t}(s)=0$. A comparison with the second part of Theorem 3.1 yields $X_{i}^{k, t}(s)=Q_{i}^{k, t}(s)$ as was to be proved.

Definition 3.4. Let $Q^{k, t}$ be the rational square matrix of order $t+1$ with $(i, s)$ entry $Q_{i}^{k, t}(s)$ for $i, s \in[0, t]$. Given any vector $\mathbf{f}^{t}=\left(f_{0}^{t}, f_{1}^{t}, \ldots, f_{t}^{t}\right)$ in $\mathbb{R}^{t+1}$, the dual Hahn transform of $\mathbf{f}^{t}$, of type $(k, t)$, is defined as the vector $\hat{\mathbf{f}}^{t}=\mathbf{f}^{t} Q^{k, t}$. In other terms, $\hat{\mathbf{f}}^{t}=\left(\hat{f}^{t}(0), \hat{f}^{t}(1), \ldots, \hat{f}^{t}(t)\right)$ where $\hat{f}^{t}(s)$ is the value at point $s$ of the dual Hahn transform polynomial

$$
\hat{f}^{t}(z)=\sum_{i=0}^{t} f_{i}^{t} Q_{i}^{k, t}(z)
$$

\section{4. $\boldsymbol{\beta}$-REGULARITY AND $T$-DESIGNS}

This section contains some further results about $T$-designs, introduced in [3]. In particular, we shall discover a close connection between the $T$-design concept and the $t$-form concept.

Definition 4.1. Given a design vector $\boldsymbol{\beta}$ in $\mathbb{R} \mathscr{P} k$, a subspace $\Phi$ of $\mathbb{R} \mathscr{P} k$ is $\boldsymbol{\beta}$-regular if and only if

$$
\langle\boldsymbol{\beta}, \phi\rangle=\frac{\langle\boldsymbol{\beta}, \mathbf{1}\rangle}{\langle\mathbf{1}, \mathbf{1}\rangle}\langle\mathbf{1}, \phi\rangle \text { for all } \phi \in \Phi .
$$

Let $T$ be a subset of the integer interval $[0, k]$. A vector $\boldsymbol{\beta} \in \mathbb{R} \mathscr{P}^{k}$ is a $T$-design vector for the Johnson scheme $J(v, k)$ if and only if

$$
\langle\boldsymbol{\beta}, \boldsymbol{\phi}\rangle=0 \text { for all } \boldsymbol{\phi} \in \operatorname{Harm}_{s}^{k} \text { with } 0 \neq s \in T \text {. }
$$


Let $\Phi$ be an $S_{v}$-invariant subspace of $\mathbb{R} \mathscr{P}^{k}$. Then $\Phi$ decomposes as an orthogonal sum $\Phi=\bigoplus_{s \in T} \operatorname{Harm}_{s}^{k}$ for a well-determined subset $T$ of $[0, k]$. This set $T$ will be referred to as the harmonic support of $\Phi$.

The $\boldsymbol{\beta}$-regularity property means that $\boldsymbol{\beta}$ behaves like the all-one vector $\mathbf{1}$ with respect to the considered "test space" $\Phi$. When $\boldsymbol{\beta}$ is the characteristic vector of a subset $\mathfrak{B}$ of $\mathbb{R}^{k}$, our $\boldsymbol{\beta}$-regularity concept reduces to the $\mathfrak{B}$ regularity introduced in [2]. Note that $\mathfrak{B}$ is a $t$-design if and only if the space $\mathrm{Hom}_{t}^{k}$ is $\mathfrak{B}$-regular.

Let us now introduce the important notion of the inner distribution [3, 4], with a suitable normalization.

Definition 4.2. The inner distribution of a design vector $\boldsymbol{\beta} \in \mathbb{R} \mathscr{P} k$ is the $(k+$ 1)-vector $\mathbf{b}^{k}=\left(b_{0}^{k}, b_{1}^{k}, \ldots, b_{k}^{k}\right)$ with

$$
\left(\begin{array}{c}
k \\
i
\end{array}\right)\left(\begin{array}{c}
v-k \\
i
\end{array}\right) b_{i}^{k}=\left\langle\boldsymbol{\beta}, C_{i}^{k, k} \boldsymbol{\beta}\right\rangle .
$$

(If $\boldsymbol{\beta}$ is the characteristic vector of a set $\mathfrak{B}$, then the right-hand side counts the pairs of blocks in $\mathfrak{B}$ that have $k-i$ points in common.)

Consider the dual Hahn transform $\hat{\mathbf{b}}^{k}=\mathbf{b}^{k} Q^{k, k}$, of spherical type $(k, k)$, of the inner distribution $\mathbf{b}^{k}$ (see Definition 3.4). By specializing Theorem 3.2 to the spherical case $\left(C_{0}^{k, k}=I\right)$, and by using the orthogonality relation in Theorem 3.1, we obtain the formula

$$
\frac{v-2 s+1}{v-s+1}\left(\begin{array}{l}
v \\
s
\end{array}\right) \hat{b}^{k}(s)=\left(\begin{array}{l}
v \\
k
\end{array}\right)\left\langle\boldsymbol{\beta}, E_{s}^{k} \boldsymbol{\beta}\right\rangle .
$$

We now are in a position to give two characterizations of the $T$-design property, in terms of the inner distribution [3] and in terms of the $\boldsymbol{\beta}$-regularity concept [2], respectively.

Theorem 4.1. (i) The vector $\boldsymbol{\beta}$ is a T-design vector if and only if the dual Hahn transform of its inner distribution satisfies $\hat{b}^{k}(s)=0$ for all $s \in T \backslash\{0\}$. (ii) The vector $\boldsymbol{\beta}$ is a $T$-design vector if and only if the $S_{v}$-invariant subspace of $\mathbb{R} \mathscr{P} k$ with harmonic support $T$ is $\boldsymbol{\beta}$-regular.

Proof. Note first that $\left\langle\boldsymbol{\beta}, E_{s}^{k} \boldsymbol{\beta}\right\rangle=\left\|E_{s}^{k} \boldsymbol{\beta}\right\|^{2}$, with $E_{s}^{k} \boldsymbol{\beta}$ the orthogonal projection of $\boldsymbol{\beta}$ in $\operatorname{Harm}_{s}^{k}$. Hence the formula above yields $\hat{b}^{k}(s) \geq 0$, for all $s \in[0, k]$, and $\hat{b}^{k}(s)=0$ if and only if $\boldsymbol{\beta}$ is orthogonal to $\mathrm{Harm}_{s}^{k}$. This proves the first statement. The second part is immediate (from Definition 4.1), since $\langle\mathbf{1}, \phi\rangle=0$ for every $\phi \in \operatorname{Harm}_{s}^{k}$ with $s \geq 1$.

We now point out an interesting application of Lemma 3.2 concerning the relationship between $T$-design vectors for two different Johnson schemes (with the same point set).

Proposition 4.1. If $\boldsymbol{\beta}$ is a $T$-design vector for $J(v, k)$, then $\boldsymbol{\beta} C_{0}^{k, t}$ is a $(T \cap$ $[0, t])$-design vector for $J(v, t)$. Conversely, if $\gamma$ is a $T$-design vector for $J(v, t)$, then $\gamma\left(C_{0}^{k, t}\right)^{T}$ is a $(T \cup[t+1, k])$-design vector for $J(v, k)$. 
We now come back to the $t$-form concept (Definition 2.2). Let $\mathbf{f}^{t} \in \mathbb{R}^{t+1}$. With each $t$-subset $x^{t}$ of $V$ we associate the vector $\xi^{t}$ (in $\mathbb{R}^{k}$ ) defined by

$$
\left(\begin{array}{l}
v-t \\
k-t
\end{array}\right) \boldsymbol{\xi}^{t}=\sum_{i=0}^{t} f_{i}^{t}\left(C_{i}^{k, t}\left(\cdot, x^{t}\right)\right)^{T}
$$

where $C_{i}^{k, t}\left(\cdot, x^{t}\right)$ denotes the $x^{t}$-column of the adjacency matrix $C_{i}^{k, t}$. It follows from Lemma 3.1 that $\xi^{t}$ belongs to the homogeneous space $\mathrm{Hom}_{t}^{k}$ of degree $t$. Let $\Phi\left(\mathbf{f}^{t}\right)$ be the subspace of $\operatorname{Hom}_{t}^{k}$ generated by the vectors $\xi^{t}$ with $x^{t} \in \mathscr{P}^{t}$. It is obvious that $\Phi\left(\mathbf{f}^{t}\right)$ is $S_{v}$-invariant.

By definition, $\mathbf{f}^{t}$ is a $t$-form for $\boldsymbol{\beta}$ if and only if the inner product $\left\langle\boldsymbol{\beta}, \boldsymbol{\xi}^{t}\right\rangle$ is independent of the choice of $x^{t}$. This means that $\Phi\left(\mathbf{f}^{t}\right)$ is a $\boldsymbol{\beta}$-regular space (see Definition 4.1). It follows from Theorem 4.1 that $\mathbf{f}^{t}$ is a $t$-form for $\boldsymbol{\beta}$ if and only if $\boldsymbol{\beta}$ is an $S\left(\mathbf{f}^{t}\right)$-design vector, where $S\left(\mathbf{f}^{t}\right)$ denotes the harmonic support of $\Phi\left(\mathbf{f}^{t}\right)$.

To identify this set $S\left(\mathbf{f}^{t}\right)$ we shall obtain another " $T$-design characterization" of the $t$-form property. We argue as follows. From a given $\boldsymbol{\beta}$ we construct the vectors $\gamma_{s}^{t}=\boldsymbol{\beta} E_{s}^{k} C_{0}^{k, t}$ in $\mathbb{R} \mathscr{P} t$, for $s \in[0, t]$. We have $\boldsymbol{\beta} E_{s}^{k} \in \operatorname{Harm}_{s}^{k}$, whence $\gamma_{s}^{t} \in \operatorname{Harm}_{s}^{t}$ by Lemma 3.2. Since $D_{i}^{t}=\sum_{s=0}^{t} Q_{i}^{k, t}(s) \gamma_{s}^{t}$, by Theorem 3.2, we observe that $\mathbf{f}^{t}$ is a $t$-form for $\boldsymbol{\beta}$ if and only if its dual Hahn transform $\hat{\mathbf{f}}^{t}=\mathbf{f}^{t} Q^{k, t}$ satisfies the equation $\sum_{s=0}^{t} \hat{f}^{t}(s) \gamma_{s}^{t}=c \mathbf{1}$, with $c \in \mathbb{R}$. This amounts to $\hat{f}^{t}(s) \gamma_{s}^{t}=\mathbf{0}$ for each $s \in[1, t]$. Since $\gamma_{s}^{t}=\mathbf{0}$ if and only if $\boldsymbol{\beta} E_{s}^{k}=\mathbf{0}$, this means that $\boldsymbol{\beta}$ is orthogonal to $\operatorname{Harm}_{s}^{k}$ for every $s$ in $[1, t]$ that satisfies $\hat{f}^{t}(s) \neq 0$. As a conclusion we obtain the following result.

Theorem 4.2. For any vector $\mathbf{f}^{t} \in \mathbb{R}^{t+1}$, let $T\left(\mathbf{f}^{t}\right)$ be the set containing the indices of the nonzero components of its dual Hahn transform $\hat{\mathbf{f}}^{t}=\mathbf{f}^{t} Q^{k, t}$, that is,

$$
T\left(\mathbf{f}^{t}\right):=\left\{s \in[0, t] \hat{f}^{t}(s) \neq 0\right\} .
$$

(i) The set $T\left(\mathbf{f}^{t}\right)$ equals the harmonic support $S\left(\mathbf{f}^{t}\right)$ of the $S_{v}$-invariant subspace $\boldsymbol{\Phi}\left(\mathbf{f}^{t}\right)$ of Hom $_{t}^{k}$ generated by the vectors $\xi^{t}$ with $x^{t} \in \mathscr{P} t$. (ii) The vector $\mathbf{f}^{t}$ is a t-form for a given $\boldsymbol{\beta}$ in $\mathbb{R}^{k}$ if and only if $\boldsymbol{\beta}$ is a $T\left(\mathbf{f}^{t}\right)$-design vector or, equivalently, if and only if the space $\Phi\left(\mathbf{f}^{t}\right)$ is $\boldsymbol{\beta}$-regular.

Proof. The essential part of the argument was given above. It remains only to show that we have $0 \in S\left(\mathbf{f}^{t}\right)$ if and only if $0 \in T\left(\mathbf{f}^{t}\right)$. This follows from the identity $\left\langle\mathbf{1}, \boldsymbol{\xi}^{t}\right\rangle=\hat{f}^{t}(0)$, which is easily proved from the definition of $\boldsymbol{\xi}^{t}$.

\section{STRUCTURE OF The $t$-FORM SPACE}

In this section, it is shown how the $t$-form space of a design vector $\boldsymbol{\beta}$ can be characterized in terms of the inner distribution $\mathbf{b}^{k}$ of $\boldsymbol{\beta}$ (Theorems 5.1 and 5.3), and how the $t$-distribution matrix of $\boldsymbol{\beta}$ can be determined from its first $r$ rows, with $r$ denoting the $t$-degree of $\boldsymbol{\beta}$ (Theorem 5.2). 
Definition 5.1. The $t$-annihilator set $A^{t}$ for $\boldsymbol{\beta}$ is defined from the dual Hahn transform $\hat{\mathbf{b}}^{k}=\mathbf{b}^{k} Q^{k, k}$ of $\mathbf{b}^{k}$ by

$$
A^{t}:=\left\{s \in[1, t] \hat{b}^{k}(s) \neq 0\right\} .
$$

The $t$-annihilator polynomial $\hat{g}^{t}(z)$ for $\boldsymbol{\beta}$, of degree $\left|A^{t}\right|$ in the variable

$$
\lambda^{t}(z)=t(v-t)-z(v+1-z)
$$

is defined by

$$
\hat{g}^{t}(z)=\prod_{s \in A^{t}}\left(\lambda^{t}(z)-\lambda^{t}(s)\right)
$$

Theorem 5.1. The $t$-degree $r$ of a design vector $\boldsymbol{\beta}$ is equal to the cardinality of the annihilator set $A^{t}$ for $\boldsymbol{\beta}$. The t-form space $\mathscr{F} t$ of $\boldsymbol{\beta}$ consists of the vectors $\mathbf{f}^{t}$ in $\mathbb{R}^{t+1}$ that satisfy $\left(\mathbf{f}^{t} Q^{k, t}\right)(s)=0$ for all $s \in A^{t}$. Equivalently, $\mathbf{f}^{t}$ belongs to $\mathscr{F}^{t}$ if and only if its dual Hahn transform polynomial $\hat{f}^{t}(z)=\sum_{i=0}^{t} f_{i}^{t} Q_{i}^{k, t}(z)$ is divisible by the t-annihilator polynomial $\hat{g}^{t}(z)$ for $\boldsymbol{\beta}$.

Proof. From Theorems 4.1 and 4.2 it follows that $\mathbf{f}^{t}$ is a $t$-form for $\boldsymbol{\beta}$ if and only if $\hat{f}^{t}(s) \hat{b}^{k}(s)=0$ for all $s \in[1, t]$, i.e., if and only if $\hat{f}^{t}(s)=0$ for all $s \in A^{t}$. This means that $\hat{f}^{t}(z)$ is divisible by $\hat{g}^{t}(z)$.

Theorem 5.1 tells us that the dual Hahn transform image of the space $\mathscr{F}^{t}$ is an ideal of the algebra of real polynomials in the variable $\lambda^{t}(z)$, reduced modulo $m^{t}(z)=\prod_{s=0}^{t}\left(\lambda^{t}(z)-\lambda^{t}(s)\right)$. This ideal contains a unique monic polynomial of minimum degree, namely the $t$-annihilator $\hat{g}^{t}(z)$. Note that $\hat{g}^{t}(z)$ divides the modulus polynomial $m^{t}(z)$. (In that respect, the only restriction is $\hat{g}^{t}(0) \neq 0$.) Conversely, any ideal of this type is the dual Hahn transform image of every design vector $\boldsymbol{\beta}$ that satisfies the condition $\hat{b}^{k}(s)=0$ if and only if $\hat{g}^{t}(s) \neq 0$ for $s \in[1, t]$.

Definition 5.2. Let $\hat{g}^{t}(z)$ be the $t$-annihilator polynomial for a given design vector $\boldsymbol{\beta}$. Consider the dual Hahn expansion $\hat{g}^{t}(z)=\sum_{i=0}^{t} g_{i}^{t} Q_{i}^{k, t}(z)$, with $g_{r}^{t} \neq 0$ and $g_{i}^{t}=0$ for $i \in[r+1, t]$. The real vector $\mathbf{g}^{t}=\left(g_{i}^{t}\right)_{i=0}^{t}$ thus defined will be called the generator $t$-form for $\boldsymbol{\beta}$ (in agreement with Theorem 5.1).

The following theorem is an extension of a known result concerning the outer distribution matrix [3] to the more general concept of the $t$-distribution matrix $D^{t}$ (see Definition 2.1).

Theorem 5.2. Let $r$ be the $t$-degree of a given design vector $\boldsymbol{\beta}$. For each $i \in[r, t]$, the row $D_{i}^{t}$ of the $t$-distribution matrix of $\boldsymbol{\beta}$ is a rational linear combination of the $r+1$ vectors $\langle\boldsymbol{\beta}, \mathbf{1}\rangle \mathbf{1}, D_{0}^{t}, \ldots, D_{r-1}^{t}$, the coefficients of which can be calculated from the annihilator set for $\boldsymbol{\beta}$.

Proof. Consider the generator $t$-form $\mathbf{g}^{t}$. In view of Definition 2.2 and Lemma 2.1, the row $D_{r}^{t}$ of $D^{t}$ can be expressed as follows:

$$
D_{r}^{t}=\left(g_{r}^{t}\right)^{-1}\left[\left(\begin{array}{l}
v \\
t
\end{array}\right)^{-1}\left(\begin{array}{l}
k \\
t
\end{array}\right) \hat{g}^{t}(0)\langle\boldsymbol{\beta}, \mathbf{1}\rangle \mathbf{1}-\sum_{i=0}^{r-1} g_{i}^{t} D_{i}^{t}\right]
$$


We can obtain a similar result for the remaining rows of $D^{t}$ by considering "shifted versions" $\left(\lambda^{t}(z)\right)^{j} \hat{g}^{t}(z)$ of the $t$-annihilator polynomial, with $j \in$ $[1, t-r]$. Details are omitted.

Remark 5.1. We briefly mention a combinatorial application. Let $\mathfrak{B}$ be a nonempty subset of $\mathscr{P}^{k}$. Define the $t$-covering radius of $\mathfrak{B}$ as the maximum for $x^{t} \in \mathscr{P}^{t}$ of the minimum for $x^{k} \in \mathfrak{B}$ of the integer $t-\left|x^{k} \cap x^{t}\right|$. It follows from Theorem 5.2 that the $t$-covering radius of $\mathfrak{B}$ is bounded from above by the $t$-ciegree of (the characteristic vector of) $\mathfrak{B}$. The reason is that the assumption $D_{0}^{t}\left(x^{t}\right)=\cdots=D_{r}^{t}\left(x^{t}\right)=0$, for a given $x^{t}$, leads to a contradiction (when $\langle\boldsymbol{\beta}, \mathbf{1}\rangle \neq 0$ ).

It is interesting to see how the structure of the $t$-form space can be derived in a purely combinatorial framework, without explicit reference to the dual Hahn polynomials. Consider the $(t+1) \times(t+1)$ tridiagonal matrix $W^{k, t}=$ $\left[w_{i, j}^{k, t}\right]_{i, j=0}^{t}$ constructed from the intersection numbers $w_{i, j}^{k, t}$ met in the proof of Theorem 3.2. By use of Definition 2.1 we obtain $W^{k, t} D^{t}=D^{t} C_{1}^{t, t}$. If $\mathbf{f}^{t}$ is a $t$-form for $\boldsymbol{\beta}$, i.e., if $\mathbf{f}^{t} D^{t}=c \mathbf{1}$, then this identity shows that $\mathbf{f}^{t} W^{k, t}$ also is a $t$-form for $\boldsymbol{\beta}$. Therefore, $W^{k, t}$ is an endomorphism of the $t$-form space of any design vector $\boldsymbol{\beta}$. In fact, we can state the following result, which is equivalent to Theorem 5.1. (The proof is omitted; see the comments below.)

Theorem 5.3. Let $W^{k, t}=\left[w_{i, j}^{k, t}\right]_{i, j=0}^{t}$. The $t$-form space $\mathscr{F}^{t}$ is $W^{k, t}$-invariant. More precisely, if $\boldsymbol{g}^{t}$ is the generator $t$-form for $\boldsymbol{\beta}$, then the $t+1-r$ vectors $\boldsymbol{g}^{t}\left(W^{k, t}\right)^{j}$ with $j \in[0, t-r]$ form a basis of $\mathscr{F}^{t}$.

The connection between the settings of Theorems 5.1 and 5.3 can be described as follows. The recurrence relation for dual Hahn polynomials (Theorem 3.1) amounts to the matrix identity $W^{k, t} Q^{k, t}=Q^{k, t} \Lambda^{t}$, with $\Lambda^{t}=$ $\operatorname{diag}\left(\lambda^{t}(0), \ldots, \lambda^{t}(t)\right)$. Consider the $W^{k, t}$-image $\mathbf{f}^{t} W^{k, t}$ of a given vector $\mathbf{f}^{t}$ in $\mathbb{R}^{t+1}$. In view of the identity above, the dual Hahn transform of this image vector equals $\hat{\mathbf{f}}^{t} \Lambda^{t}$, and the associated polynomial is the product $\hat{f}^{t}(z) \lambda^{t}(z)$ reduced modulo $m^{t}(z)$.

Remark 5.2. It is possible to work out the theory of $t$-forms for any value of $t$ with $0 \leq t \leq\lfloor v / 2\rfloor$. Let us briefly examine the case $t \geq k$ (instead of $t \leq k$ as above). In this situation, we have to replace $t$ by $k$ in the definition of the adjacency matrices, roughly speaking. Thus, the $t$-form space $\mathscr{F}^{t}$ of a design vector $\boldsymbol{\beta}$ in $\mathbb{R}^{k}$ now is a subspace of $\mathbb{R}^{k+1}$. It can be verified that the $t$-degree of $\boldsymbol{\beta}$ is independent of $t$, and counts the integers $s \in[1, k]$ that satisfy $\hat{b}^{k}(s) \neq 0$. The dual Hahn transform image of the $t$-form space of $\boldsymbol{\beta}$ is the same for all $t$ (in the range $k \leq t \leq\lfloor v / 2\rfloor$ ). The appropriate transform is given by the polynomials $Q_{i}^{t, k}(z)$ with $i \in[0, k]$. This can be proved from the preceding results by use of the fact that the $t$-form space of $\boldsymbol{\beta}$ coincides with the $k$-form space of $\boldsymbol{\beta}\left(C_{0}^{t, k}\right)^{T}$, a vector in $\mathbb{R} \mathscr{P} t$.

Example 5.1. Let $\boldsymbol{\beta}$ be the design vector for the Steiner system $S(5,8,24)$, that is, the unique 5-design $\mathfrak{B}$ of index 1 with $v=24, k=8$, and $|\mathfrak{B}|=$ 759. Its inner distribution vector (Definition 3.4) is well known and easily calculated as $\mathbf{b}^{8}=759(1,0,0,0,280,0,448,0,30)$. From Definition 3.4, 
the components of the dual Hahn transform $\hat{\mathbf{b}}^{8}=\mathbf{b}^{8} Q^{8,8}$ of $\mathbf{b}^{8}$ are $\hat{\mathbf{b}}^{8}(0)=$ $(3.11 .23)^{2}, \hat{\mathbf{b}}^{8}(6)=(8.9 .17 .23) / 13, \hat{\mathbf{b}}^{8}(8)=5.8 .23$, and $\hat{\mathbf{b}}^{8}(i)=0$ for $i=$ $1,2,3,4,5,7$. So from Theorem $4.1, \boldsymbol{\beta}$ is a $T$-design vector for any $T \subseteq$ $\{0,1,2,3,4,5,7\}$, and in particular, is a 5-design. From Definition 5.1, $A^{8}=\{6,8\}, A^{7}=A^{6}=\{6\}$, and $A^{t}=\varnothing$ for $0 \leq t \leq 5$. Hence from Definition 2.2 the respective $t$-degrees are $r^{8}=2, r^{7}=r^{6}=1$, and $r^{t}=0$ for $0 \leq t \leq 5$. For $t=6,7,8$ the $t$-annihilator polynomials of type $(8, t)$ are, from Definitions 5.1, 5.2, and 3.3,

$$
\begin{gathered}
\hat{g}^{6}(z)=(6-z)(19-z)=18 Q_{0}^{8,6}(z)+3 Q_{1}^{8,6}(z), \\
\hat{g}^{7}(z)=(6-z)(19-z)=2 Q_{0}^{8,7}(z)+2 Q_{1}^{8,7}(z), \\
\hat{g}^{8}(z)=(6-z)(19-z)(8-z)(17-z)=16 Q_{0}^{8,8}(z)+16 Q_{1}^{8,8}(z)+4 Q_{2}^{8,8}(z) .
\end{gathered}
$$

From Definition 2.2, the generator $t$-form identities directly deduced from these are:

$$
\begin{gathered}
6 D_{0}^{6}\left(x^{6}\right)+D_{1}^{6}\left(x^{6}\right)=6, \\
D_{0}^{7}\left(x^{7}\right)+D_{1}^{7}\left(x^{7}\right)=1, \\
4 D_{0}^{8}\left(x^{8}\right)+4 D_{1}^{8}\left(x^{8}\right)+D_{2}^{8}\left(x^{8}\right)=4,
\end{gathered}
$$

for every $x^{t} \in \mathscr{P}^{t}$. Note that the three equations above have 2,2 , and 3 nonnegative integer solutions respectively, namely $(1,0)$ or $(0,6),(1,0)$ or $(0,1)$, and $(1,0,0),(0,1,0)$ or $(0,0,4)$. Theorem 5.2 shows how to calculate the whole $t$-distribution matrix $D^{t}$. Note that $D^{t}$ is a $(t+1) \times\left(\begin{array}{c}24 \\ t\end{array}\right)$ matrix, but by the calculations above, has only $r^{t}+1$ distinct columns! (It is interesting to note here that any of the three equations above would imply that $\mathfrak{B}$ is a Steiner system.) As in Remark 5.2, it is also possible to calculate the $t$-form space for $9 \leq t \leq 12$, with $t$-degree $r^{t}=2$. As an illustration,

$$
20 D_{0}^{12}\left(x^{12}\right)+6 D_{1}^{12}\left(x^{12}\right)+D_{2}^{12}\left(x^{12}\right)=132 .
$$

(Remember that $D_{i}^{12}\left(x^{12}\right)$ counts the blocks of $\mathfrak{B}$ that have $8-i$ points in common with a given 12-subset $x^{12}$ of the 24-set $V$.)

Example 5.2. Let us briefly examine the simple situation characterized by $r^{t}=$ 1 , i.e., by $A^{t}=\left\{s_{0}\right\}$ for some $s_{0}$ with $1 \leq s_{0} \leq t \leq k$. According to Theorem 5.1, this corresponds to $T$-design vectors $\boldsymbol{\beta}$ with $T=[1, t] \backslash\left\{s_{0}\right\}$. The $t$-annihilator polynomial is $\hat{g}^{t}(z)=\left(s_{0}-z\right)\left(v+1-s_{0}-z\right)$. From its dual Hahn expansion we obtain the following generator $t$-form identity:

$$
\begin{gathered}
{\left[s_{0}\left(v+1-s_{0}\right)-t(v-k)\right] D_{0}^{t}\left(x^{t}\right)+(k+1-t) D_{1}^{t}\left(x^{t}\right)} \\
=\left(\begin{array}{c}
v \\
t
\end{array}\right)^{-1}\left(\begin{array}{l}
k \\
t
\end{array}\right) s_{0}\left(v+1-s_{0}\right)\langle\boldsymbol{\beta}, \mathbf{1}\rangle .
\end{gathered}
$$

The first two cases of Example 5.1 above illustrate this result, with $\left(t, s_{0}\right)=$ $(6,6)$ and $(7,6)$, respectively.

Example 5.3. We now give a "combinatorial construction" in the special case $s_{0}=1$. Suppose that $\mathfrak{B}_{0}$ is a $t$-design for $J(v-1, k)$ and $\mathfrak{B}_{1}$ is a $t$-design for $J(v-1, k-1)$, with the same point set $V_{0}$ (of cardinality $v-1$ ). Consider 
the combinatorial structure in $J(v, k)$ with the point set $V=V_{0} \cup\{\infty\}$ and with the block set $\mathfrak{B}=\mathfrak{B}_{0} \cup \mathfrak{B}_{1}^{\prime}$, where $\mathfrak{B}_{1}^{\prime}$ consists of the $k$-sets $x \cup\{\infty\}$ with $x \in \mathfrak{B}_{1}$. We suppose that $\mathfrak{B}$ is not a 1-design. Then, the pair $\left(D_{0}^{t}\left(x^{t}\right), D_{1}^{t}\left(x^{t}\right)\right)$ for $\mathfrak{B}$ assumes exactly two distinct values, one value in the case $\infty \in x^{t}$ and one (different) value in the other case. Hence there exists a nontrivial identity $g_{0}^{t} D_{0}^{t}\left(x^{t}\right)+g_{1}^{t} D_{1}^{t}\left(x^{t}\right)=c$ (for all $\left.x^{t} \in \mathscr{P}^{t}\right)$. The pair $\left(g_{0}^{t}, g_{1}^{t}\right)$ is uniquely determined, within normalization, and $g_{1}^{t}$ is not zero. Elementary "design computation" yields $g_{0}^{t}=v-t(v-k)$ and $g_{1}^{t}=k+1-t$. Therefore, our construction exemplifies the situation examined above, with $s_{0}=1$. As a conclusion, $\mathfrak{B}$ is a $[2, t]$-design for $J(v, k)$, although it is not a $t$-design.

\section{ACKNOWLEDGMENT}

The authors express their gratitude to the anonymous referee whose constructive remarks led to a more compact and systematic presentation of their results.

\section{REFERENCES}

1. A. R. Calderbank and P. Delsarte, On error-correcting codes and invariant linear forms, SIAM J. Discrete Math. 6 (1993), 1-23.

2. A. R. Calderbank, P. Delsarte, and N. J. A. Sloane, A strengthening of the Assmus-Mattson theorem, IEEE Trans. Information Theory IT-37 (1991), 1261-1268.

3. P. Delsarte, An algebraic approach to the association schemes of coding theory, Philips Res. Rep. Suppl. 10 (1973).

4. _ Pairs of vectors in the space of an association scheme, Philips Res. Rep. 32 (1977), 373-411.

5. _ Hahn polynomials, discrete harmonics, and t-designs, SIAM J. Appl. Math. 34 (1978), 157-166.

6. C. F. Dunkl, Spherical functions on compact groups and applications to special functions, Sympos. Math. 22 (1977), 145-161.

7. An addition theorem for Hahn polynomials: the spherical functions, SIAM J. Math. Anal. 9 (1978), 627-637.

8. R. L. Graham, S.-Y. R. Li, and W.-C. W. Li, On the structure of $t$-designs, SIAM J. Algebraic Discrete Methods 1 (1980), 8-14.

9. J. E. Graver and W. B. Jurkat, The module structure of integral designs, J. Combin. Theory Ser. A 15 (1973), 75-90.

10. S. Karlin and J. L. McGregor, The Hahn polynomials, formulas and an application, Scripta Math. 26 (1961), 33-46.

11. D. K. Ray-Chaudhuri and N. M. Singhi, On existence of t-designs with large $v$ and $\lambda$, SIAM J. Discrete Math. 1 (1988), 98-104.

12. R. M. Wilson, Inequalities for t-designs, J. Combin. Theory Ser. A 34 (1983), 313-324.

13. _ On the theory of t-designs, Enumeration and Designs (D. M. Jackson and S. A. Vanstone, eds.), Academic Press, New York, 1984, pp. 19-49.

Mathematical Sciences Research Center, AT\&T Bell Laboratories, Murray Hill, New JERSEY 07974

E-mail address: rc@research.att.com

Department of Computer Science, University of louvain, Place Sainte Barbe 2, B 1348 Louvain La Neuve, Belgium

E-mail address: delsarte@info.ucl.ac.be 